\title{
Effectiveness of BNT162b2 Vaccine in Adolescents during Outbreak of SARS-CoV-2 Delta Variant Infection, Israel, 2021
}

Aharona Glatman-Freedman, Yael Hershkovitz, Zalman Kaufman, Rita Dichtiar, Lital Keinan-Boker, Michal Bromberg

In Israel, the BNT162b2 vaccine against severe acute respiratory syndrome coronavirus 2 was approved for use in adolescents in June 2021, shortly before an outbreak of B.1.617.2 (Delta) variant-dominant infection. We evaluated short-term vaccine effectiveness and found the vaccine to be highly effective among this population in this setting.

Tn May 2021, the US Food and Drug Administration Iand the European Medicines Agency expanded existing authorization for BNT162b2 vaccine (PfizerBioNTech, https://www.pfizer.com) against severe acute respiratory syndrome coronavirus 2 (SARSCoV-2) to include its use in adolescents $12-15$ years of age $(1,2)$. On June 2, 2021, the Israel Ministry of Health declared the availability of BNT162b2 vaccine for adolescents 12-15 years of age (3) as a 2-dose regimen, given 21 days apart. By August 26, 2021, a total of 277,218 adolescents ( $46.1 \%$ of those eligible) had received 1 dose of the vaccine and 187,707 (31.2\%) had received 2 doses (Figure 1, panel A). In mid-June 2021, after a month of extremely low SARS-CoV-2 activity in Israel, 2 local outbreaks erupted (4-6). These outbreaks marked the beginning of a new widespread SARS-CoV-2 outbreak in Israel (Figure 1, panel B), dominated by the B.1.617.2 (Delta) variant, which accounted for $93 \%-99 \%$ of the sequenced viruses during July and August 2021 (7). We analyzed effectiveness of this vaccine among adolescents who had been vaccinated in the early stages of this outbreak in Israel.

Author affiliations: The Israel Center for Disease Control,

Ramat Gan, Israel (A. Glatman-Freedman, Y. Hershkovitz,

Z. Kaufman, R. Dichtiar, L. Keinan-Boker, M. Bromberg); Tel Aviv University, Tel Aviv, Israel (A. Glatman-Freedman, M. Bromberg); Haifa University, Haifa, Israel (L. Keinan-Boker)

DOI: https://doi.org/10.3201/eid2711.211886
The study was approved by the superior ethical committee of the Israel Ministry of Health and included exemption from informed consent.

\section{The Study}

We performed a nationwide retrospective cohort study to estimate vaccine effectiveness against PCRconfirmed SARS-CoV-2 infections among adolescent Israel residents 12-15 years of age who had received the second vaccine dose during July 1-24, 2021. The data sources used are described in detail elsewhere (8). We estimated vaccine effectiveness and 95\% CIs by using ( 1 - incidence rate ratio) $\times 100$ for $1-7,8-14$, 15-21, and 22-28 days after the second vaccine dose. Incidence rate ratio denotes the ratio of the rate of PCR-confirmed SARS-CoV-2 infections in the vaccinated and unvaccinated groups.

We excluded from analysis adolescents who had had a documented SARS-CoV-2-positive PCR result before the evaluation periods, regardless of their vaccination status. When several positive SARS-CoV-2 test results were documented for the same person during the study period, we included only the first result in our analysis.

We determined the number of unvaccinated controls for each date during July 1-24, 2021, by omitting the number of fully vaccinated adolescent Israel residents $12-15$ years of age who had received the second BNT162b2 vaccine dose on a particular date from the total number of Israel residents who did not have a documented SARS-CoV-2-positive test result and had not received only a single vaccine dose by that date. We expressed the denominators of the vaccinated and unvaccinated groups in person-days.

After administration of the second vaccine dose, crude vaccine effectiveness against laboratory-confirmed SARS-CoV-2 infection was 55.3\% (95\% CI 


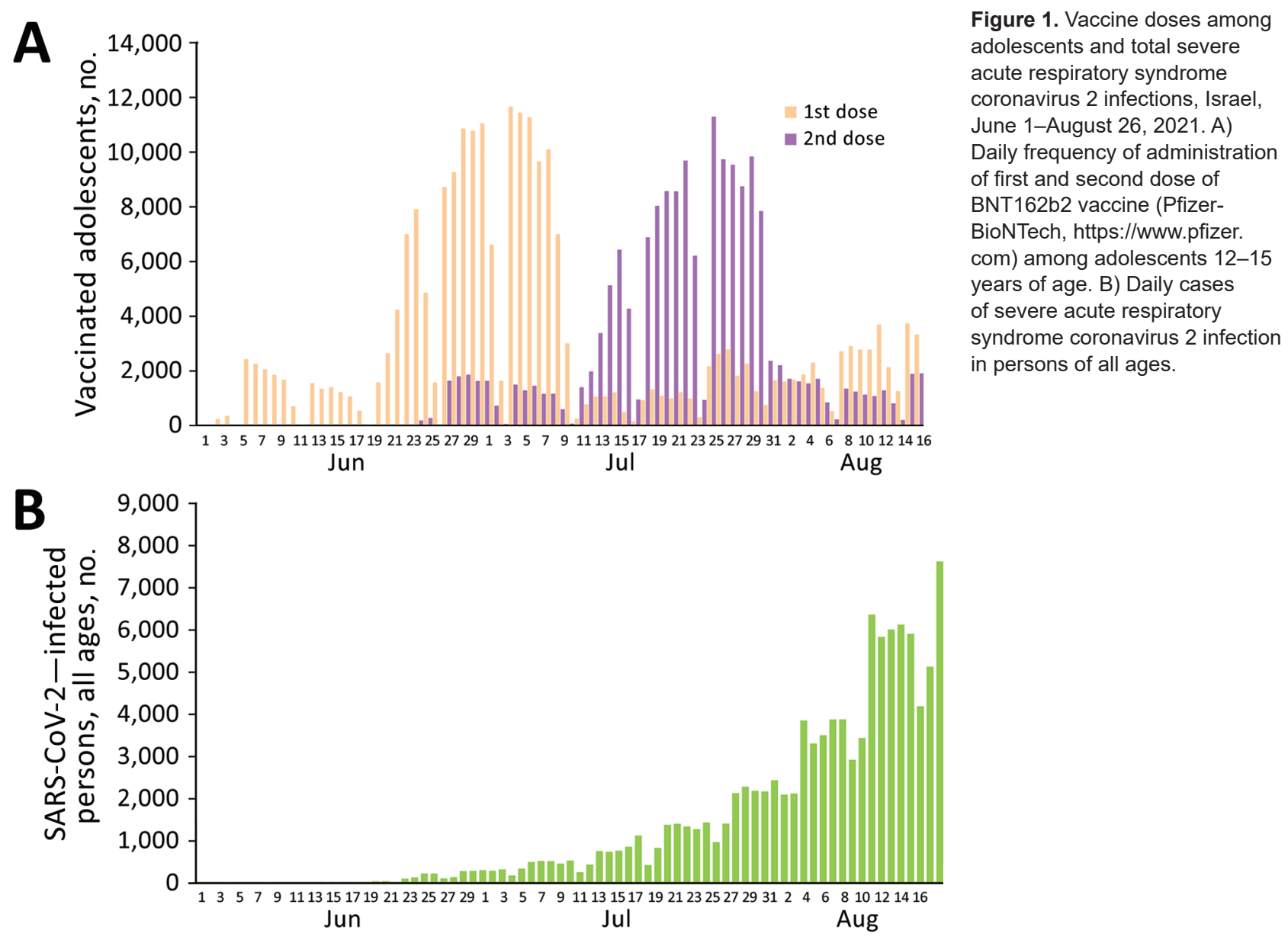

$41.3 \%-66.0 \%)$ in the first week, $87.1 \%$ (95\% CI $81.0 \%$ $91.2 \%)$ in the second week, $91.2 \%(87.4 \%-93.8 \%)$ in the third week, and $88.2 \%$ (95\% CI 85.0\%-90.7\%) in the fourth week (Table; Figure 2). Vaccine effectiveness differed significantly between the first and subsequent weeks, but we found no statistically significant differences in vaccine effectiveness among the second, third, and fourth weeks. Because of the small number of cases of SARS-CoV-2 infection among vaccinated adolescents, we could not adjust for weekly vaccine effectiveness evaluation. However, adjustments for sex and epidemiologic week for days 8-28 after the second dose combined demonstrated adjusted vaccine effectiveness of $91.5 \%$ (95\% CI $88.2 \%-93.9 \%$ ) against SARS-CoV-2 infection (Table). We did not estimate vaccine effectiveness against symptomatic diseases because epidemiologic investigation was performed for $42 \%$ of vaccinated and $40 \%$ of unvaccinated adolescents in our cohort.

As of August 26, 2021, none of the vaccinated adolescents who became SARS-CoV-2-positive on days 1-28 after the the second vaccine dose had been hospitalized. By that same date, among unvaccinated adolescents, $7(0.38 \%)$ of 1,825 who tested positive for SARS-CoV-2 on days 1-7 after vaccinated adolescents had received their second vaccine dose and $26(0.32 \%)$

Table. Effectiveness of BNT162B2 vaccine against PCR-confirmed SARS-CoV-2 infection in adolescents 12-15 years of age after receipt of second dose, Israel, $2021^{*}$

\begin{tabular}{lccccccc}
\hline Days after & $\begin{array}{c}\text { Unvaccinated, } \\
\text { SARS-CoV-2- } \\
\text { positive, no. }\end{array}$ & $\begin{array}{c}\text { Unvaccinated } \\
\text { person-days }\end{array}$ & $\begin{array}{c}\text { Vaccinated, } \\
\text { SARS-CoV-2- } \\
\text { positive, no. }\end{array}$ & $\begin{array}{c}\text { Vaccinated } \\
\text { person-days }\end{array}$ & $\begin{array}{c}\text { Crude vaccine } \\
\text { effectiveness } \\
(95 \% \text { Cl) }\end{array}$ & $\begin{array}{c}\text { Adjusted vaccine } \\
\text { effectiveness } \\
(95 \% \text { Cl) }\end{array}$ \\
\hline Week 1 & $1-7$ & 1,825 & $10,148,829$ & 53 & 673,129 & $55.3(41.3-66.0)$ & NA \\
Week 2 & $8-14$ & 2,923 & $9,750,816$ & 26 & 672,790 & $87.1(81.0-91.2)$ & NA \\
Week 3 & $15-21$ & 4,906 & $9,386,429$ & 31 & 672,624 & $91.2(87.4-93.8)$ & NA \\
Week 4 & $22-28$ & 7,510 & $8,905,457$ & 67 & 672,328 & $88.2(85.0-90.7)$ & NA \\
Weeks 2-4 & $8-28$ & 8,144 & $13,623,714$ & 124 & $2,034,591$ & $89.8(87.8-91.5)$ & $91.5(88.2-93.9)$ \\
\hline
\end{tabular}

*BNT162B2 vaccine, Pfizer-BioNTech (https://www.pfizer.com) NA, not applicable; SARS-CoV-2, severe acute respiratory syndrome coronavirus 2. 


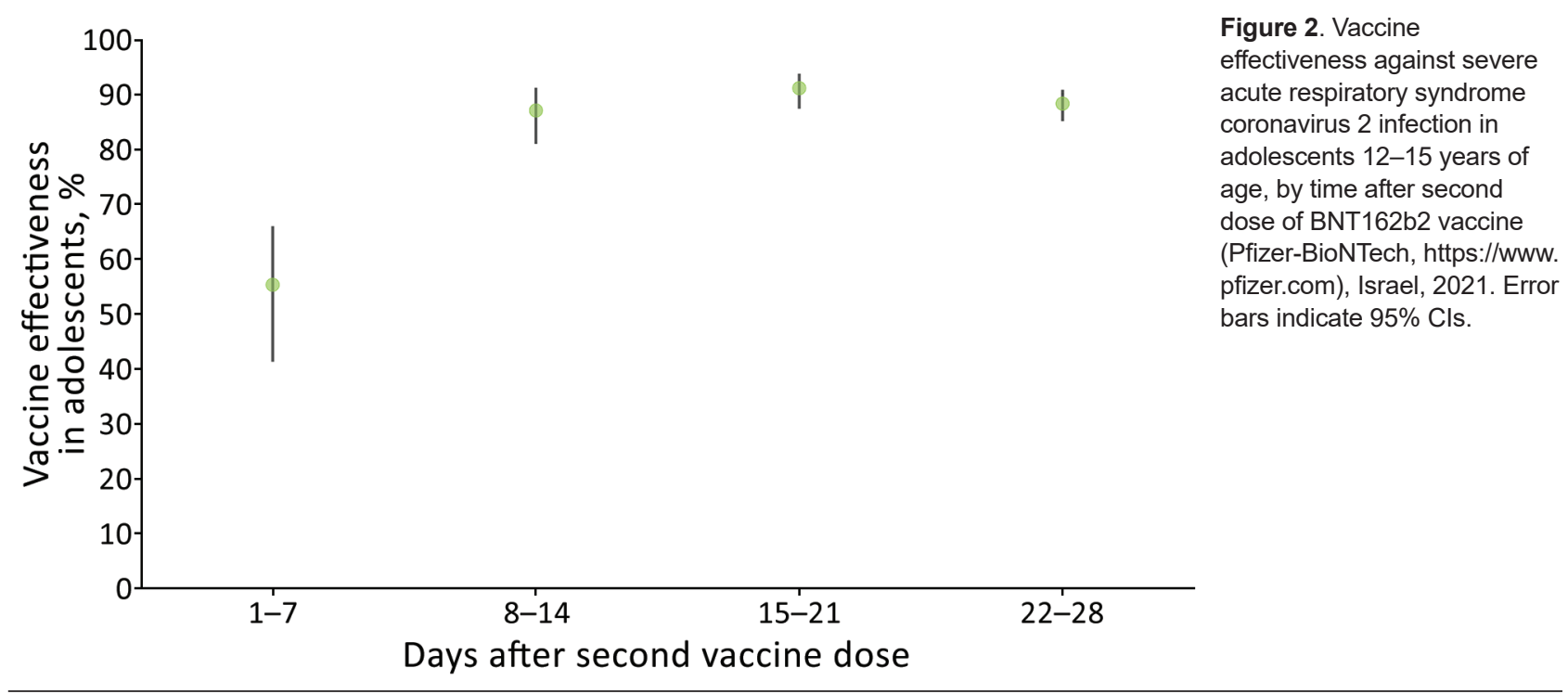

of 8,144 who tested positive on days 8-28 were hospitalized. Also by August 26, no vaccinated or unvaccinated SARS-CoV-2-positive adolescents had died.

\section{Conclusions}

The BNT162b2 vaccination campaign for adolescents 12-15 years of age in Israel coincided with the outbreak of the SARS-CoV-2 Delta variant. As such, the timing enabled estimation of vaccine effectiveness against SARS-CoV-2 infection for this age group during predominant circulation of the Delta variant.

Our results demonstrate high vaccine effectiveness against SARS-CoV-2 infection in this population starting the second week after the second vaccine dose. These estimates are somewhat lower than those that had been estimated for persons 16-39 years of age during the same time intervals after the second vaccine dose during circulation of the SARS-CoV-2 Alpha variant and wild-type virus in Israel (8). Specifically, adjusted vaccine effectiveness against SARSCoV-2 infection for persons 16-39 years of age was $93.2 \%(95 \%$ CI $91.9 \%-94.2 \%)$ at $8-14$ days, $96.7 \%$ (95\% CI 95.8\%-97.4\%) at 15-21 days, and 96.6 (95\% CI $95.7 \%-97.3 \%)$ at $22-28$ days after receipt of the second vaccine dose. Although vaccine effectiveness estimates and 95\% CIs during the circulation of the Alpha variant and the wild-type virus were adjusted for age, sex, and epidemiologic week, only minor differences in point estimates and 95\% CIs were noted between crude and adjusted vaccine effectiveness (8).

The effectiveness estimate of $55.3 \%$ in the first week after the second dose probably reflects the effect of the first vaccine dose. This estimate is consistant with previous estimates of vaccine effectiveness 14-20 days after the first dose (8-10).
Our findings are consistent with those of a recent study from the United Kingdom, which demonstrated vaccine effectiveness of $88.0 \% \quad(95 \%$ CI $85.3 \%-90.1 \%$ ) against symptomatic disease caused by the SARS-CoV-2 Delta variant (11), compared with vaccine effectiveness of 93.7\% (95\% CI 91.6\%-95.3\%) against disease caused by the Alpha variant among persons $\geq 16$ years of age who had received 2 doses of BNT162b2 (11). However, that study addressed neither the interval between the 2 doses nor the exact interval between assessment of vaccine effectiveness and the date of the second dose (11).

As of September 2021, two controlled studies had assessed vaccine efficacy in adolescents, without specifying the SARS-CoV-2 variant $(12,13)$. One study reported BNT162b2 vaccine efficacy of $100 \%$ against laboratory-confirmed COVID-19 $\geq 7$ days after receipt of the second vaccine dose at 21 days after the first dose (12). The other study reported that vaccine efficacy of the mRNA-1273 vaccine 14 days after the second dose was difficult to assess because of the low incidence of laboratory-confirmed COVID-19 in the trial population (4 cases in the placebo group and 0 cases in the mRNA-1273 group) (13). Of note, the geometric mean ratio of neutralizing antibodies in adolescents receiving those vaccines was similar to or greater than that of young adults after receipt of 2 doses $(12,13)$.

Behavioral and testing policy factors can potentially affect estimations of vaccine effectiveness. Behaviors that increase exposure to SARS-CoV-2 may be assumed by vaccinated or unvaccinated adolescents for different reasons and are difficult to measure. During the study period, SARS-CoV-2 testing was available in Israel regardless of vaccination status. 
The recent rise in SARS-CoV-2 cases in Israel raised 2 concerns. The first concern was that BNT162b2 vaccine-elicited immunity was waning. Waning of spike protein antibody levels was detected over time after receipt of a second dose of SARS-CoV-2 vaccines (14). The second concern was that the vaccine was not effective against the SARS-CoV-2 Delta variant. However, our findings indicate that the BNT162b2 vaccine provides adolescents with highly effective short-term protection against the SARS-CoV-2 Delta variant.

A.G.-F. conceived and designed the study, led data analysis, and wrote the first draft of the manuscript. M.B. oversaw the study design and analysis. Y.H. and R.D. retrieved the data. Y.H. and R.D. performed data analysis. Z.K. performed comparative evaluation with previous data. A.G.-F., Z.K., L.K.-B., and M.B. interpreted the data and edited the final manuscript. All authors revised the manuscript critically for important intellectual content and approved the final version of the manuscript.

\section{About the Author}

Dr. Glatman-Freedman is the director of the Infectious Diseases Unit in the Israel Center for Disease Control. She is affiliated academically with the School of Public Health at Tel Aviv University. Her primary research interests are the epidemiology of respiratory viruses, syndromic surveillance, and vaccine effectiveness.

\section{References}

1. Food and Drug Administration. Coronavirus (COVID-19) update: FDA authorizes Pfizer-BioNTech COVID-19 vaccine for emergency use in adolescents in another important action in fight against pandemic [cited 2021 Aug 23]. https://www. fda.gov/news-events/press-announcements/coronaviruscovid-19-update-fda-authorizes-pfizer-biontech-covid19-vaccine-emergency-use

2. European Medicines Agency. First COVID-19 vaccine approved for children aged 12 to 15 in EU [cited $2021 \mathrm{Aug}$ 23]. https:/ / www.ema.europa.eu/en/ news/first-covid-19vaccine-approved-children-aged-12-15-eu

3. Israel Ministry of Health. Ministry of Health's position regarding the expansion of the vaccination operation to ages 12-16 years [cited 2021 Aug 23]. https:/ / www.gov.il/en/ departments/news/02062021-01
4. Israel Ministry of Health. Coronavirus outbreak in Binyamina [cited 2021 Aug 23]. https:/ / www.gov.il/en/ departments/news/19062021-02

5. Israel Ministry of Health. Effective today (20.6.2021) masking requirement at all schools in Modi'in and Binyamina [cited 2021 Aug 23]. https:/ / www.gov.il/en/ departments/news/20062021-01

6. Israel Ministry of Health. Home Front Command coronavirus testing points closed [cited 2021 Aug 23]. https://www.gov.il/en/departments/news/02062021-03

7. Our World in Data. SARS-CoV-2 variants in analyzed sequences, Israel [cited 2021 Aug 18]. https:/ / ourworldindata.org/grapher/covid-variants-area?country $=\sim$ ISR

8. Glatman-Freedman A, Bromberg M, Dichtiar R, Hershkovitz Y, Keinan-Boker L. The BNT162b2 vaccine effectiveness against new COVID-19 cases and complications of breakthrough cases: a nation-wide retrospective longitudinal multiple cohort analysis using individualised data. EBioMedicine. 2021;72:103574. https:/ / doi.org/10.1016/ j.ebiom.2021.103574

9. Dagan N, Barda N, Kepten E, Miron O, Perchik S, Katz MA, et al. BNT162b2 mRNA Covid-19 vaccine in a nationwide mass vaccination setting. N Engl J Med. 2021;384:1412-23.

10. Chodick G, Tene L, Patalon T, Gazit S, Ben Tov A, Cohen D, et al. Assessment of effectiveness of 1 dose of BNT162b2 vaccine for SARS-CoV-2 infection 13 to 24 days after immunization. JAMA Netw Open. 2021;4:e2115985. https://doi.org/10.1001/jamanetworkopen.2021.15985

11. Lopez Bernal J, Andrews N, Gower C, Gallagher E, Simmons R, Thelwall S, et al. Effectiveness of Covid-19 vaccines against the B.1.617.2 (Delta) variant. N Engl J Med. 2021;385:585-94. https:/ / doi.org/10.1056/NEJMoa2108891

12. Frenck RW, Jr., Klein NP, Kitchin N, Gurtman A, Absalon J, Lockhart S, et al. Safety, immunogenicity, and efficacy of the BNT162b2 Covid-19 vaccine in adolescents. N Engl J Med. 2021;385:239-50.

13. Ali K, Berman G, Zhou H, Deng W, Faughnan V, Coronado-Voges M, et al. Evaluation of mRNA-1273 SARS-CoV-2 vaccine in adolescents. N Engl J Med. 2021 Aug 11 [Epub ahead of print]. https:// doi.org/10.1056/ NEJMoa2109522

14. Shrotri M, Navaratnam AMD, Nguyen V, Byrne $T$, Geismar C, Fragaszy E, et al.; Virus Watch Collaborative. Spike-antibody waning after second dose of BNT162b2 or ChAdOx1. Lancet. 2021;398:385-7. https://doi.org/10.1016/ S0140-6736(21)01642-1

Address for correspondence: Aharona Glatman-Freedman, The Israel Center for Disease Control, Israel Ministry of Health Gertner Institute Building, Tel Hashomer, Ramat Gan 5265601, Israel; email: aharona.freedman@moh.gov.il 